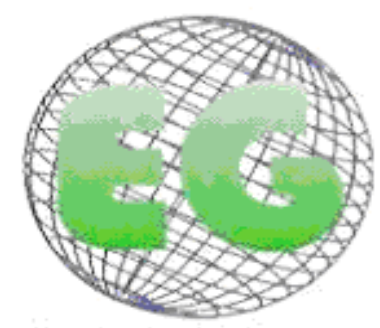

ISSN 1695-6141 N'25.
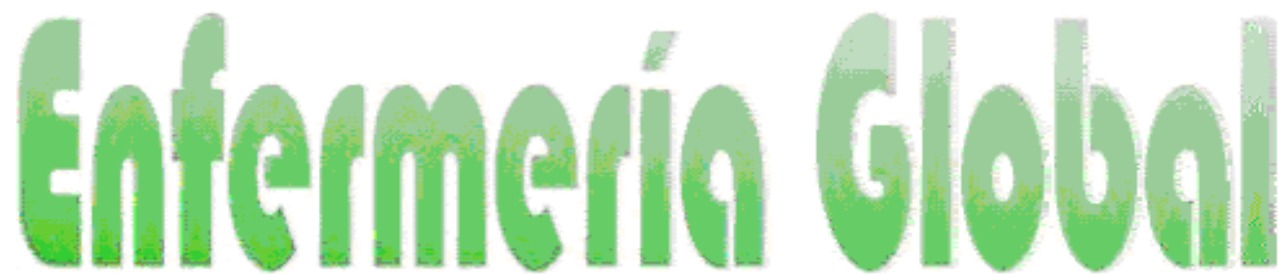

Revista electrónica trimestral de Enfermería

Enero 2012

www.um.es/egloball

\title{
Influencia de la interdisciplinaridad en las acciones del enfermero en Hemovigilancia
}

A interdisciplinaridade influenciando nas ações do enfermeiro em Hemovigilância

\begin{abstract}
*Dias, MAM., "*Viana, LO.
*Mestre em Enfermagem pela Escola de Enfermagem Anna Nery (EEAN/UFRJ); Membro-Fundadora do Núcleo de Educação e Saúde em Enfermagem (NUPESENF)do Departamento de Metodologia da EEAN/UFRJ; Enfermeira do Serviço de Educação Continuada do Hospital dos Servidores do Estado (HSE/RJ). "**Prof ${ }^{a}$ Dra $^{a}$ Titular do Departamento de Metodologia da EEAN/UFRJ. Membro do (NUPESENF). Brasil.
\end{abstract}

\begin{abstract}
Palabras clave: vigilancia sanitaria; recursos humanos en salud; competencia profesional; educación en enfermería; ética profesional
\end{abstract}

Palavras-chave: vigilância sanitária; recursos humanos em saúde; competência profisional; educação em enfermagem; ética profissional

Keywords: health surveillance; health manpower; professional competence; professional ethics

\section{RESUMEN}

Este artículo proviene de parte de uno de los capítulos de análisis de la tesis de maestría en enfermería que tiene como tema la formación y la capacitación de las enfermeras en hospitales centinela de hemovigilancia en Río de Janeiro. Este nuevo frente en Vigilancia Sanitaria destaca a la Enfermera como la desencadenadora de las acciones de investigación específicas por ser el profesional que más eventos adversos testimonia en su práctica diaria. Tiene como objeto las relaciones profesionales de hemovigilancia y objetiva analizar la práctica cotidiana de las enfermeras frente a la transdisciplinariedad. Concluye que el alcance profesional de la enfermera profesional se ha ampliado, y requiere de otros profesionales el respeto y el reconocimiento de sus acciones en la inversión de las nuevas fronteras de la salud.

\section{RESUMO}

Este artigo provém de parte de um dos capítulos de análise de dissertação de mestrado em enfermagem que traz como temática a formação e competências do enfermeiro em Hemovigilância nos Hospitais Sentinela do Município do Rio de Janeiro. Esta nova frente em Vigilância Sanitária destaca o Enfermeiro como o deflagrador das ações de investigação específica, por ser o profissional que mais testemunha eventos adversos no cotidiano de sua prática. Tem como objeto as relações profissionais em Hemovigilância e objetiva analisar o cotidiano da prática do enfermeiro frente a transdisciplinaridade. Conclui-se que a abrangência profissional do 
Enfermeiro se expandiu, exigindo assim, dos outros profissionais envolvidos, respeito e reconhecimento de suas ações no investimento de novas fronteiras em saúde.

\begin{abstract}
This article comes from part of one of the chapters of analysis in a master's thesis in nursing whose subject is the training and skills of nurses in Haemovigilance Sentinel Hospitals in Rio de Janeiro. This new perspective in Health Surveillance highlights nursing as the trigger for actions for specific research since the nurses are theprofessionals who witness most adverse events in their daily practice. The object of the study is the professional relations in Haemovigilance and the it analyzes the everyday practice of nurses in the light if interdisciplinarity. The study concludes that the professional scope of the nurse has broadened and demands the respect and recognition of the nurse's actions on the part of other professionals in the new frontiers of health.
\end{abstract}

\title{
INTRODUCCIÓN
}

A partir del proyecto instituido por la Agencia Nacional de Vigilancia Sanitaria (Anvisa) bajo la tutela del Ministerio de Salud, el Proyecto Hospitales Centinela tiene como objetivo construir una red de hospitales en todo el país, preparados para notificar eventos adversos y quejas técnicas de productos de salud, insumos, materiales y medicamentos, saneantes, y equipamiento médico en uso en Brasil. ${ }^{1}$ Con este fin, se establecieron tres instrumentos de investigación de salud: la Tecnovigilancia (detección de eventos adversos com materiales médico-hospitalarios); la Farmacovigilancia (detección de eventos adversos con fármacos) y la Hemovigilancia, que ocurre cuando la adversidad proviene de factores imunológicos a partir de la administración de hemocomponentes con la consecuente identificación de una reación transfusional, que puede ocurrir imediata o tardíamente a la infusión de eritrocitos, plaquetas, plasma, crioprecipitados o hemoderivados.

\section{METODOLOGÍA}

De los 182 hospitales denominados Centinelas en el territorio nacional en 2009, diez pertenecían al Municipio do Rio de Janeiro de los cuales ocho aceptaron participar en la presente investigación. Tras la aprobación de los referidos Comités de Ética y Pesquisa (CEP) de las instituciones, el enfermero de cada hospital responsable de la notificación en Hemovigilancia, tras la firma del TCLE grabó entrevistas en aparatos MP4 las cuales se analizaron a la luz de Philippe Perrenoud ${ }^{2}$, sociólogo francés cuyas obras abarcan conceptualizaciones relativas a la competencia profesional. Los sujetos fueron nombrados con títulos de nobleza y las instituiciones recibieron nombres de castillos, debido a la nobleza de la sangre, en tanto que líquido único e insustituible en el cuerpo de los seres humanos.

Toda nueva actividad propuesta para el equipo de enfermería toca el gran problema del cuadro de personal que, generalmente, está siempre por debajo de la cuota requerida para la atención de las demandas de la asistencia. Este hecho es de suma importancia, pues la cantidad de personal de enfermería está directamente relacionada con la calidad de la asistencia prestada en la prevención de factores de riesgo para la clientela. En este punto, se enfatiza la composición del equipo multidisciplinar en Hemovigilancia, la autonomía del enfermero ante este equipo y la actuación del médico en el momento de los eventos de transfusión, como factores de gran importancia. 
Los datos colectados se analizaron a la luz de los conceptos de competencia de Philippe Perrenoud, sociólogo suizo, profesor de la Facultad de Psicología y Ciencias de la Educación de la Universidad de Ginebra en su publicación "10 Nuevas Competencias para Enseñar." (2000)²

\section{RESULTADOS}

\section{Composición del Equipo en Hemovigilancia}

La cuestión recursos humanos fue trabajada por la Enfermera Duquesa del Castillo de Alcobaça, con recursos de la propia ANVISA a partir de la contrapartida monetaria que este órgano propone invertir en las instituciones centinela. La enfermera aclara:

Porque normalmente los alumnos de enfermería identifican, dentro de la universidad, proyectos en los que ellos pueden participar. Esas dos entraron en el proyecto y nosotros lo enviamos a Anvisa y conseguimos bolsa. Así ellas quedaron durante dos años como bolsistas. Sólo que una perdió la bolsa y la otra se trasladó. (DUQUESA)

Esta iniciativa comprueba que, a partir de $\mathrm{ANVISA}^{3}$, hay posibilidades de invertir en iniciación científica y contribuir en recursos humanos de las Instituiciones Centinela y en la formación de los futuros profesionales.

En el Castillo Thor, la Hemovigilancia no tiene un proceso continuo debido a la escasez de enfermeros. Observemos el siguiente relato:

Hay varios momentos en que yo no tengo personal. Tuve una enfermera con permiso de gestación, una jubilación y quedamos sin hacer Hemovigilancia durante un buen tiempo. Cuando yo tengo personal yo hago, cuando no tengo, no hay cómo hacer. Entonces, simplemente, no se consigue hacer Hemovigilancia cuando se tiene a alguien ausente, licenciado o de vacaciones. No hay una regularidad hasta el momento. (EMPERATRIZ)

Ya en el Castillo de los Mouros, las enfermeras del servicio de hemoterapia no se involucran con la Hemovigilancia. Estas profesionales realizan el examen clínico de los donantes, atienden complicaciones en la sala de recogida de sangre y actúan en procedimientos de aféresis realizadas en el propio servicio. La Enfermera Archiduquesa comenta sobre ellas:

Ellas pertenecen a la División de Enfermería, mas toda su jerarquía es dirigida por la jefa médica de servicio. La médica tiene que autorizar, vamos a decirlo así. (ARCHIDUQUESA)

La jerarquía del equipo de enfermería está inserta en el ejercicio de la profesión influyendo sobre la autonomía de las acciones del enfermero que tiene la dirección de las divisiones de enfermería en los Hospitales Centinela. Luego, la presentación de estos profesionales a otras categorías, atenta contra nuestro Código de Ética de Enfermería. 


\title{
Autonomía del Equipo de Enfermería en Hemovigilancia
}

La participación jerárquica de las enfermeras del servicio de hemoterapia es diferente dentro de cualquier equipo de enfermería del Castillo de Mouros. Se percibe por el testimonio de la enfermera Archiduquesa, que las mismas mostraron cierta resistencia a colaborar en la formación continuada del equipo de enfermería de la unidad hospitalaria, cuando fueron solicitadas y la garantía de la presencia de la médica es referida por la propia división de enfermería, como "la jefa" de esas enfermeras:

\begin{abstract}
Percibimos su capacitación, lo que ellas tienen de conocimiento desde el primer momento en que fueron invitadas, y ahí la División de Enfermería hizo una presión mayor: - Y vaya, que lo hace, estará con nosotros junto con la Gerente de Riesgo y vamos a contar con la presencia de la jefa de ustedes, jefa médica. (ARCHIDUQUESA)
\end{abstract}

Este conflicto de gestión se refleja en el desinterés de esas enfermeras en la inversión científica del equipo de enfermería de la institución, pues se sienten comprometidas exclusivamente con los intereses específicos del servicio de hemoterapia y no del hospital como un todo, como revela la Enfermera Archiduquesa:

\section{$Y$ sin embargo, no hemos visto interés. Si hubiese interés, ellas se enfrentarían. Intenté estimularlas: - Vamos a hacer un trabajo en ese nivel, un trabajo científico. - Ah, no, no tengo voluntad. Mis trabajos científicos ya se hacen dentro del servicio, orientados a él. (ARCHIDUQUESA)}

Es notoria la resistencia de las enfermeras de hemoterapia en relación a la influencia jerárquica de la dirección de enfermería en este hospital ante la imposición de la jefa médica, pues la enfermera Archiduquesa comenta: "Ahí nosotros vimos que tienen un potencial increiblel. Una de ellas sabe transmitir bien el conocimiento. Mas no consigo liberarlas."

En la mayoría de los hospitales las transfusiones son realizadas por el equipo que presta asistencia directa al paciente. En dos de las ocho instituciones abordadas, el servicio de hemoterapia no tiene profesional de enfermería en su cuadro de funcionarios. Este hecho puede comprobarse por todos estos testimonios:

Aquí en el Castillo de Sesimbra no tenemos un equipo responsable de la transfusión. No hay un equipo de transfusiones en el sector, que sería lo ideal. (PRINCESA)

El profesional de enfermería que recibe la bolsa de sangre para transfundir realiza la transfusión en el sector. (ARCHIDUQUESA)

El equipo de transfusión es el equipo de enfermería del hospital. El equipo de enfermería bajo la coordinación del enfermero. Todavía no tenemos un equipo centralizado de transfusión. (DUQUESA)

El profesional de la agencia de transfusiones llega con la sangre $o$ el hemoderivado del paciente para ser transfundido, lo entrega en 
la clínica [...] Coloca el sello, entrega la sangre y el enfermero se hace responsable de su administración. (VIZCONDESSA)

En el Castillo Mafra, único de la Red Centinela del municipio de Rio de Janeiro que desde hace cinco años tiene un equipo de enfermería transfusional oficializada, reconocida y respetada por todos los profesionales del hospital, el director de enfermería salió del servicio de hemoterapia por solicitud de la jefa médica del sector, permaneciendo todos los profesionales de enfermería subordinados a la dirección médica. Este tipo de conducta abarca factores éticos que también implican el código de conducta de nuestra profesión. Sin duda, este hecho repercute en el desempeño de las acciones de enfermería de este grupo, que está siendo coordinado por un profesional cuya formación no consiste en conocimientos específicos de asistencia de enfermería.

Tosoli y Oliveira ${ }^{4}$ relacionan autonomía con formación cuando afirman:

En este proceso de conquista de la autonomía, se citan diversas dificultades, entre las que destacamos la formación profesional no fomentadora de una práctica autónoma y la ausencia de especificidad del papel propio como generadora de limitaciones en el ejercicio de una práctica autónoma. (TOSOLI; OLIVEIRA, 2005)

Al tratar sobre problemas éticos de la profesión, Perrenoud ${ }^{2}$ explica que la violencia no es sólo golpes, lesiones, robo y vandalismo. Es el ataque a la libertad de expresión, de movimiento, de comportamiento. Siempre que el enfermero vea lesionada su actuación por un miembro del equipo de salud de otra categoría, queda expresa la amenaza velada que la presencia permanente del equipo de enfermería representa en la institución.

Esta amenaza se relaciona con el hecho de que el número de profesionales de enfermería en una institución de salud es bastante mayor que el de cualquier otra categoría profesional del área. Luego, una decisión tomada por todo el equipo de enfermería de un hospital tendría, ciertamente, una significativa repercusión social y política dentro de la institución. Si esa decisión fuese asumida por los equipos de enfermería de los varios segmentos institucionales, tal progresión se volvería realmente amenazadora, afectando al status quo de la salud en el país.

¿Será que nuestro equipo ya se dio cuenta de esto? ¿Somo conscientes de la fuerza, y consecuentemente, del poder que impone nuestro conocimiento a partir de un trabajo ininterrumpido de la categoría con mayor número de representantes en cualquer unidad de salud? El conocimientpo en enfermería garantiza nuestro poder y certifica nuestro saber, pues, el saber engendra poder.

Sin embargo, con la autonomía de enfermería del Castillo Mafra visiblemente debilitada, la Hemovigilancia continúa siendo realizada por un enfermero que, a pesar de que también desempeña la función de selector de donantes, es el Hemovigilante y emite su opinión:

Luego, dentro de un gran hospital que trabaja con sangre el equipo de transfusión es reciente, a pesar de que fue un hito trabajar con un equipo sólo para eso. Me parece importante que en todas las unidades que trabajan con sangre, siempre que haya un paciente para transfundir, debería haber alguien responsable para la transfusión. (RAJÁ) 
Cincidimos con el Enfermero Rajá al referirse al Equipo de Enfermería de Transfusiones del Castillo Mafra como um "hito" en el desempeño de enfermería en Hemovigilancia. Tiene que haber, en los administradores de enfermería, una posición resolutiva en el tema de los recursos humanos para que se cumpla la fiabilidad de la obligación de notificación. Nombrar e invertir en la formación de las enfermeras para llevar a cabo actividades relacionadas con los eventos centinela en la transfusión, demuestra en la división de enfermería del Castillo Mafra, una madurez en la comprensión de nuevas habilidades en la práctica de enfermería en la vigilancia de la salud.

Para una efectiva transcendencia del objetivo mayor del enfermero - promoción del cuidado con calidad y seguridad - la organización del trabajo necesita de metas que vayan más allá de lo convencional e invierta en nuevas direcciones, en busca de la excelencia por medios todavía considerados escépticos, en probar lo inédito, en la busca de lo ideal, traspasando inclusive, las barreras de la transdisciplinaridad. Instaurar un equipo de profesionales de enfermería en transfusión sale al encuentro de la especificidad de la propuesta del Proyecto Centinela fortificando las bases de la investigación en Hemovigilancia.

La presencia de un equipo de enfermería de transfusión activa la busca de eventos adversos, pues, al final de cada transfusión de hemocomponente, el transfusor retorna al cliente para comprobar los signos vitales y evaluar con el paciente y el equipo asistencial la ocurrencia de algun tipo de evento referente a la transfusión tomando, entonces, las medidas aceptables para la investigación. Esta acción la hace el Hemovigilante de la institución Centinela.

El equipo de enfermería transfusional es un "sueño" que la mayoría desea alcanzar, pero en el Castillo Pessegueiro la enfermera entrevistada confiesa no haber pensado en esa posibilidad. Sobre la idea de tener un equipo específico de enfermería para trabajar en transfusión, ella relata:

Yo formo parte del cuadro de profesionales, todavía no tratan de dar la debida importancia a formar un equipo sólo para vigilar este procedimiento. [...] Yo planeo; si ya tuviese, de repente, un conocimiento más profundo de la necesidad, ya habría hecho un plan orientado a ello. Aunque fuese a medio plazo. (VIZCONDESA)

Perrenoud ${ }^{2}$ define equipo como un "grupo reunido en torno a un proyecto común, cuya realización pasa por diversas formas de acuerdo y de cooperación." Afirma (Op. cit.) que un equipo reunido para llevar adelante una empresa definida puede, al final, involucrarse en una nueva aventura y crear una red permanente de cooperación. En cuanto a conflictos existentes entre equipos él sugiere: "En cuanto dejemos de demonizar el conflicto, consideremoslo como un componente de la acción colectiva y pregúntemonos cómo podemos utilizarlo de manera más constructiva que destructiva" ${ }^{2}$.

Tanto los ejemplos del Castillo de Alcobaça, en relación a la adquisición de bolsistas en la difusión del saber, como el Castillo Mafra, manteniendo un equipo de enfermería de transfusión, es probable que sean seguidos por otros Hospitales Centinela tanto en la difusión del conocimiento como en el apoyo a la eficacia de investigación en la transfusión apoyando las investigaciones que, así, tendrán mayor garantía de prevención en la transgresión de los factores éticos en transfusión. 


\section{Actuación del médico junto al equipo de enfermería em Hemovigilancia}

La composición del equipo de Hemovigilancia en los Hospitales Centinela, en la actualidad, puede ser analizada por los siguientes testimonios:

Mi equipo de Hemovigilancia está compuesto por un médico que es del servicio de hemoterapia y está participando en evaluar algunas reacciones [...] y una bióloga, que es responsable, por ejemplo, identificó una reacción de la transfusión: ¿De cuándo es la muestra? ¿Hizo prueba cruzada de nuevo? ¿El PAl (Investigación de Anticuerpos Irregulares) después de este paciente fue negativo? ¿Hubo error? ¿Repite la prueba cruzada? Así la bióloga se responsabiliza de esto. (DUQUESA)

Hicimos una reunión y básicamente yo hice una propuesta de montar un equipo. Estaban presentes las enfermeras del servicio, médicos del servicio de hemoterapia, intenté esbozar personalmente con las personas presentes de hacer parte de un equipo para trabajar una propuesta. Una médica, una enfermera y una técnica estarían conmigo. [...] En julio de 2006 recibimos una médica que quería trabajar en sistema de guardia y la dirección la envió a Hemoterapia y esta, a su vez, llegó a asumir la Hemovigilancia (ARCHIDUQUESA)

Quien hace la busca activa son tres enfermeros. Los responsables de la Hemovigilancia son un enfermero y un médico. Trabajan conjuntamente y toman las decisiones. (PRINCESA)

Entonces la médica de hemoterapia va a la cama y conversa con este paciente para ver si él presentó alguna reacción. (MARQUESA)

Aqui en el hospital tenemos un equipo de transfusión. [...] Tenemos también una médica que es del equipo. Yo siempre paso consulta con ella, cuando hay dudas conversamos sobre el asunto y uno ayuda al otro. Discutimos el asunto y llegamos a la conclusión de cuál fue el tipo de reacción. (RAJÁ)

Dentro de la Hemovigilancia nosotros tenemos específicamente una médica que está haciendo esto. Si hay transfusión ella va hasta allí, directamente a la cama; con esto se ha mejorado. (VIZCONDESA)

Él es bioquímico y trabaja más de 20 años en esta parte de sangre. Fue del INCA y está unos tres años en él y sustituyó en esta parte de la agencia de transfusión. (CONDESA)

Con todo, cuando analizamos la conducta de los médicos en la actuación directa con el paciente en el momento de las reacciones transfusionales, tenemos los siguientes relatos:

Si hubiera médico; la gran preocupación de todos cuando comenzamos a divulgar la importancia de la reacción de la 
transfusión era quién va a atender. [...] Nunca se definió en la institución la posibilidad de la existencia del médico al lado del enfermero durante una reacción. (ARCHIDUQUESA)

Porque muchas veces se constató que los médicos estaban lejos de lo que estaba sucediendo en la parte transfusional. (PRINCESA)

Porque la médica es rotativa. Entonces, en el tiempo de que dispone, no consigue hacer lo que ella acredita. Ella tiene experiencia de otro hospital, entonces ella lo intenta. Intentó implementar, pero no tiene tiempo para hacer esto. (VIZCONDESA)

Yo creo que cuando la ficha de la transfusión no es cumplimentada por el médico que visualizó la reacción es un problema crítico en todo el proceso. (RAJÁ)

Eran buenos médicos, pero no estaban muy preocupados con los resultados. (DUQUESA)

Sólo actúa si hay alguna reacción. Si no hubiera reacción alguna, él solo va a ver el prontuario por la mañana. Piden hematócrito y hemoglobina y se acabó. Yo no veo participación del equipo médico, no, muy al contrario. (CONDESA)

Esa falta de participación del médico en el momento de la reación de la transfusión tal vez pueda explicarse por el conocimiento al respecto del asunto. Durante la formación continuada sobre el tema el equipo médico no concretiza la intención de promover la difusión de este enfoque, lo que se observa a partir de los testimonios:

Había una propuesta de irnos a la residencia médica y pasarnos a Hemovigilancia, mas nunca conseguimos avanzar. (ARCHIDUQUESA)

Es lo que ellos también están queriendo hacer; una cosa más sistematizada con los otros médicos, con los anestesistas. Porque, así, como en la facultad de enfermería esto es tratado muy superficialmente, en la facultad de medicina también es superficial. Entonces tienen que tener un conocimiento más exhaustivo, más profundo sobre varios aspectos, por ser una ciencia nueva. (PRINCESA)

En el Castillo Pessegueiro, la enfermera Vizcondesa dice que la formación de las enfermeras de las clínicas se realiza por las dos médicas hematologas de la agencia de transfusión de la institución, las cuales no dirigieron ningún tipo de encuentro para aclarar a los médicos del hospital sobre el asunto. Incluso en relación con lo que se hace con el equipo de enfermería, Vizcondesa comenta que "No existe específicamente una preocupación de aplicar, incluso de profundizar en el conocimiento en Hemovigilancia."

Por eso, de alguna manera, existe la preocupación por la formación en enfermería: 
Colocamos esto en la posgraduación y en todos los cursos. También tenemos aqui dos cursos al año para los técnicos de enfermería. Entonces siempre se coloca la cuestión de la Hemovigilancia, pero relacionada con el impacto de observación de reacciones. (VIZCONDESA)

Perrenoud ${ }^{2}$ aclara que un proyecto de formación continua puede reforzar una cultura de cooperación. Según el autor (op. cit.) el proceso de validación de conocimientos experienciales amplía gradualmente el círculo de los profesionales capaces de autoevaluar sus competencias.

\section{DISCUSIÓN}

La interdisciplinaridad es imperativa en relación a la Hemovigilancia, siendo necesario que los profesionales formen una red de conocimientos, colaboración y solidaridad en favor de la investigación de eventos adversos en transfusión, lo que traerá beneficios a los pacientes y mérito a la institución. La Bioética es un campo interdisciplinar, en la medida en que congrega esfuerzos de profesionales y pensadores de todas las áreas del conocimiento ${ }^{5}$. En este sentido la bioética puede definirse como un estudio interdisciplinar, ligado a la ética, que investiga, em el área de las ciencias de la vida y de la salud la totalidad de las condiciones necesarias para una administración responsable de la vida humana en general y de la persona humana en particular 6 .

Así, la autonomía profesional de los enfermeros y la vulnerabilidad de los pacientes en relación a eventos de transfusión, indican que el item referente a la composición del equipo en la Hemovigilancia está inserto en la $7^{\underline{a}}$ competencia de Perrenoud (involucrar a los pares), cuando contempla graduandos en iniciación científica y cuando compone un equipo específico para actuar en Hemovigilancia.

La inserción de futuros profesionales sustenta la continuidad de las acciones en Hemovigilancia y amplía la gama de opciones de estos enfermeros, en el futuro, en cuanto a su actuación en el mercado de trabajo, a partir de una experiencia solamente vivida en instituciones Centinela. De la misma forma, la constitución de un equipo de enfermería de transfusiones, dinamiza el proceso del acto de la transfusión brindando la oportunidad de hacer notificaciones fieles a los datos reales respecto a las normas bioéticas.

Se refiere también la 6a competencia (participar de la administración de la escuela), sustituyéndose el escenario de la escuela por el hospital, en el momento en que se reúne con otros segmentos para providenciar la implantación de la Hemovigilancia en la institución. Perrenoud $^{2}$ en la competencia supracitada alerta de que decidir colectivamente es asumir, también, los errores de estrategia.

Cuando el enfermero se reúne con otros profesionales para definir equipo y normas de implantación de la Hemovigilancia, trasciende su campo de acción y asume una postura interdisciplinar dentro del equipo de salud. Compartir responsabilidades refleja madurez y conquista en el campo de la acción.

La 9a competencia: enfrentar los deberes y los dilemas éticos de la profesión, está expresada em el ítem "autonomía del equipo de enfermería en Hemovigilancia", al hablar de discriminación en la escuela. La formación pasa por el conjunto del currículo y por una práctica reflexiva de los valores a infundir ${ }^{2}$. En la enseñanza de graduación de todas las categorías en salud, el respeto a los otros profesionales y el valor de sus competencias, 
debería ser un tema a explorar y enfatizado en disciplina referente al ejercício profesional, con la intención de prevenir futuros embates que perjudican, en última instancia, al cliente.

La 5 $5^{\text {a }}$ competencia de Perrenoud es trabajar en equipo, que está integralmente relacionada con esta unidad temática. Al profundizar la discusión sobre la unión entre profesionales en la realización de un proyecto, el autor especifica que:

Cada uno de los participantes tiene interés en que su equipo funcione, mas, a veces, es involucrado en cuestiones de poder, en proyectos a defender, en juegos de relación o emociones que provocan divergencia, incertidumbre o desorden en el funcionamiento colectivo?

La interdisciplinaridad es una condición que deberá ser conquistada paulatinamente por los profesionales. La constatación del saber que el enfermero demuestre en Hemovigilancia traerá a los demás profesionales de salud implicados en esta temática, la convicción de que aquel espacio es realmente para ser ocupado por él. Este enfermero deberá mantener viva la llama de la formación permanente y promover condiciones de enseñanza-aprendizaje como un vínculo entre los profesionales de la asistencia presentando los resultados de la inversión del equipo de enfermería frente a la Hemovigilancia.

En cuanto a la participación del médico en el momento del evento de la transfusión al discurrir sobre el conjunto de situaciones complejas, prácticas y problemas profesionales en la competencia referente al trabajo en equipo, Perrenoud advierte:

El verdadero trabajo de equipo comienza cuando los miembros se alejan del "muro de las lamentaciones" para actuar, utilizando toda la zona de autonomía disponible y toda la capacidad de negociación de un actor colectivo que está determinado, para realizar su proyecto, a apartar las restricciones institucionales y a obtener los recursos y los apoyos necesarios ${ }^{2}$.

El equipo de enfermería debe señalar, a través de relatos de eventualidades, la ausencia del médico en el momento de las complicaciones de la transfusión y, a su vez, la gerencia de enfermería del sector y la dirección Del equipo llevar el hecho, de manera oficial, a las instancias relacionadas com la resolución. La insistencia en este tipo de posicionamiento en el equipo de enfermería evidenciará determinación en la intencionalidad de la calidad de la asistencia y, para ello, la necesidad de un trabajo interdisciplinar. La interdisciplinaridad actualmente es objeto de estudio muy destacado que tropieza en la autonomíay hegemonía del otro cuando se relacionan los equipos de enfermería y médico.

En cuanto hubiera un paciente internado en una clínica, por lo menos un representante de cada uno de esos equipos debe estar presente, independientemente del estado de riesgo del paciente. Esta situación abarca cuestiones legales del ejercicio de la profesión y la omisión del equipo de enfermería en señalar formalmente la ausencia del profesional médico, es conveniente caso de que no haya registro que compruebe la solicitud de la presencia del médico. Esta es una cuestión que en Hospitales Centinela debe ser dirigida al Comité de Transfusión para las debidas providencias.

Los relatos de enfermería, en ese sentido, se basan a partir del Capítulo 3 del Código de Ética Médica ${ }^{7}$ que en su Artículo 8ำ determina que "está prohibido al médico ausentarse de sus actividades profesionales, incluso temporalmente, sin dejar a otro médico encargado de la atención a sus pacientes ingresados o en estado grave". 
Para apoyar aún más la postura del enfermero ante la ausencia del médico durante una guardia, puede citarse la Sección 1 del Código de Ética Profesional de Enfermería ${ }^{8}$, que trata de responsabilidades y deberes del enfermero. En su redación, se lee en el Artícuo 21: "Es responsabilidad del enfermero proteger a la persona, familiar o colectividad contra daños derivados de imprudencia, negligencia o impericia por parte de cualquier miembro del equipo de salud".

Perrenoud ${ }^{2}$ al referirse a las reglas de vida común y sanciones deduce que, contrariamente a lo que se imagina, a veces, la negociación no conduce en modo alguno al poço rigor, pues, cuando las reglas son adoptadas por el grupo, se imponen a todos, y cada uno se vuelve avalista de su ejecución. Por tanto, la presencia del médico tanto como la del enfermero, no debe negligenciarse cuando se trata de proceso de transfusión.

En el servicio de hematología, el hematologo debe tener una presencia diurna, así como los servicios médicos y la enfermera, pues todos están bajo juramento componiendo el equipo de salud. Cualquier actitud en contrario, atenta contra el código de ética de ambas profesiones, sujetos a las sanciones civiles y penales, pues abarca situaciones de negligencia y omisión de socorro ${ }^{9}$.

Chavez ${ }^{10}$ y colaboradores afirman que el área de gerencia en salud ha sido históricamente incorporada como función del Enfermero, quien debe estar adecuadamente preparado para las constantes y cambios de los perfiles de gestión. Los autores complementan también que otras características se exigen a los gestores, como flexibilidad, capacidad de relación y valentía para enfrentar nuevos desafíos. Específicamente la gestión de personas, en busca constante de la calidad y excelencia en los servicios, han provocado cambios significativos en esta área, buscando invertir cada vez más en los individuos y en las relaciones humanas.

En este momento Perrenoud 2 nos advierte de que un trabajo de alto riesgo exige que las personas se involucren sin abusar de su poder. Luego, es evidente que el respeto, el reconocimiento y la colaboración de los profesionales en servicios de salud son factores esenciales en el alcance de la interdisciplinaridad.

\section{CONCLUSIONES}

El Proyecto Hospitales Centinela trajo al enfermero una serie de nuevas competencias que le destacan ante eventos adversos por ser el profesional que más los testimonia en el cotidiano de su práctica por ser esta presencial y continua en relación al paciente. Por tanto, a partir de las competencias referidas por Perrenoud ${ }^{2}$, se puede atribuir al enfermero en Hemovigilância ${ }^{10}$ :

- Estimular y promover estrategias y condiciones del trabajo interdisciplinar:

- Participar en reuniones y promover, en la institución, eventos referentes a la Hemovigilancia;

- Informar e involucrar a todo el equipo asistencial en la rutina de la transfusión;

- Enfrentar y proponer soluciones para los dilemas éticos de la profesión relacionados con la Hemovigilancia. 


\section{REFERÊNCIAS}

1. Brasil, Ministério da Saúde. Agência Nacional de Vigilância Sanitária (Anvisa). Projetos Hospitais Sentinela: uma estratégia de vigilância para a póscomercialização de produtos de saúde. Brasília, 2001. Disponível em http://www.anvisa.gov.br/servicosaude/hsentinela/historico.htm Acesso em 22/10/2006

2. Perrenoud, P. 10 Novas Competências para Ensinar. Tradução Patrícia Chittoni Ramos. Porto Alegre:SC. Artmed, 2000

3. Brasil, Ministério da Saúde. Agência Nacional de Vigilância Sanitária - Anvisa. Termos de Referência - Serviços de Saúde Sentinela: estratégia para a vigilância de produtos pós-comercializados. Grupo 2. 2001. Disponível em http://www.anvisa.gov.br. Acesso em maio/2008

4. Tosoli, MA; Oliveira, DC. The social representation of nurse's professional autonomy im public health. Revista Brasileira de Enfermagem, v.58,n4, p. 393-398, 2005

5. Cohen,C; Salgado, MTM. Reflexão sobre a autonomia civil das pessoas portadoras de transtornos mentais. Revista de Bioética., v 17, no 2 Brasília, Conselho Federal de Medicina, 2009 (p.222)

6. Loureiro, CRM. Introdução ao Biodireito. S.ao Paulo: Saraiva, 2009

7. Brasil, Conselho Regional de Medicina do Rio de Janeiro (CRM/RJ) Resolução CRM no 1931/2009. Código de Ética Médica. Disponível em http://www.gineco.com.br Acesso em 11/10/2009

8. Brasil, Conselho Federal De Enfermagem. Resolução COFEN № 311/2001: Código de Ética Profissional de Enfermagem. Disponível em http://www.portalcofen.br. Acesso em 11/10/1009

9. Dias, MAM; Viana, LO. O Enfermeiro na Hemovigilância: sua formação e competências. Dissertação de Mestrado. Ewcola de Enfermagem Anna Nery, UFRJ, 2009

10.Chavez, MM; Menezes Brito, MJ; Cozer Montenegro, L; Alves, M. competências profesionales de los enfermeros: El método developing a curriculum como posibilidad para elaborar um projecto pedagógico. Enfermería Global. № 18, febrero, 2010. Disponível em www.um.es/eglobal// 